\title{
Inhibitory effects of Lactobacillus casei subsp. rhamnosus on Salmonella lipopolysaccharide- induced inflammation and epithelial barrier dysfunction in a co-culture model using Caco-2/ peripheral blood mononuclear cells
}

\author{
Hsu-Wei Fang, ${ }^{1,2} \dagger$ Shiuh-Bin Fang, ${ }^{3} \dagger$ Jen-Shiu Chiang Chiau, ${ }^{4}$ \\ Chun-Yan Yeung, ${ }^{5,6}$ Wai-Tao Chan, ${ }^{5}$ Chuen-Bin Jiang, ${ }^{5}$ Mei-Lien Cheng ${ }^{4}$ \\ and Hung-Chang Lee 5,7
}

\begin{abstract}
Correspondence Hung-Chang Lee ped2435@ms2.mmh.org.tw
\end{abstract}

Received 27 January 2009 Accepted 23 January 2010

\footnotetext{
${ }^{1}$ Department of Chemical Engineering and Biotechnology, National Taipei University Technology, Taipei, Taiwan, ROC

${ }^{2}$ Division of Medical Engineering Research, National Health Research Institutes, Miaoli, Taiwan, $\mathrm{ROC}$

${ }^{3}$ Centre for Paediatric Gastroenterology, Royal Free Campus, University College London Medical School, London, UK

${ }^{4}$ Department of Medical Research, Mackay Memorial Hospital, Taipei, Taiwan, ROC

${ }^{5}$ Department of Paediatrics, Mackay Memorial Hospital, Taipei, Taiwan, ROC

${ }^{6}$ Mackay Medicine, Nursing and Management College, Taipei, Taiwan, ROC

${ }^{7}$ Department of Paediatrics, Taipei Medical University, Taipei, Taiwan, ROC
}

In this study, we investigated the anti-inflammatory and reinforcing barrier effects of Lactobacillus casei subsp. rhamnosus (Lcr35) on Caco-2 intestinal epithelial cells already exposed to Salmonella LPS. Using the Transwell co-culture model, Salmonella LPS was apically added to polarized Caco-2 cells co-cultured with peripheral blood mononuclear cells (PBMCs) in the basolateral compartment. LPS-stimulated Caco-2 cells were incubated with Lcr35 for 1, 6, 24 or $48 \mathrm{~h}$. Apical inoculation of Lcr35 after $48 \mathrm{~h}$ significantly inhibited the basolateral secretion of interleukin-8 (IL-8) in the Caco-2/PBMC co-culture. The PCR analysis showed that Lcr35 significantly downregulated mRNA expression of monocyte chemoattractant protein 1 (MCP-1) $(P<0.05)$ and had a trend of decreasing mRNA expression of IL-8 $(P=0.05)$, but did not alter mRNA expression of transforming growth factor- $\beta 1$ in LPS-stimulated Caco- 2 cells at $48 \mathrm{~h}$ after addition of Lcr35. Compared to non-LPS-pretreated controls, transepithelial electrical resistance (TEER) of the polarized Caco-2 cell monolayers pretreated with LPS for $48 \mathrm{~h}$ was decreased by $9.9 \%(P<0.05)$. Additionally, compared to those cells only treated with LPS, apical co-incubation with Lcr35 showed biphasic TEER levels increased by $12.1 \%(P<0.001), 5.7 \%(P<0.05)$ and $86.8 \%(P<0.001)$ in the Caco-2 cell monolayers compared to those without Lcr35 treatment after 1, 6 and $48 \mathrm{~h}$, respectively. In conclusion, Lcr35 can exert anti-inflammatory effects and ameliorate barrier dysfunction in the Salmonella LPS-pretreated inflamed intestinal epithelium in vitro.
†These authors contributed equally to this work.

Abbreviations: IEC, intestinal epithelial cell; IL-8, interleukin-8; MCP-1, monocyte chemoattractant protein 1 ; NF- $\kappa \mathrm{B}$, nuclear factor-kappa $\mathrm{B}$; PBMC, peripheral blood mononuclear cell; TEER, transepithelial electrical resistance; TGF- $\beta 1$, transforming growth factor- $\beta 1$; TNF- $\alpha$, tumour necrosis factor alpha.

\section{INTRODUCTION}

The intestinal epithelium maintains a physical barrier to separate the luminal environment from the host milieu, and acts as a guard of the intestinal tract resisting bacteria, pathogens and other antigens (Sierro et al., 2001). Such intestinal barrier integrity can stabilize the entire intestinal 
ecosystem. In the maintenance of barrier integrity, tight junction complexes play a decisive role (Mitic et al., 2000). Intestinal infections by microbial pathogens, such as Salmonella spp., often impair or subvert host tight junction barrier function and can subsequently result in acute diarrhoea. Moreover, certain enteric viruses and bacteria can cause deterioration in the structure and function of intestinal tight junctions that may lead to chronic intestinal disorders (O’Hara \& Buret, 2008).

In addition to behaving as a physical barrier, the intestinal epithelium also plays a key role in the host immune system. Various lymphocyte populations interspersed among the epithelial cells or in the underlying intestinal lamina propria constitute the gut-associated lymphoid tissue to provide innate and adaptive immune responses against pathogens (Ramiro-Puig et al., 2008). Accordingly, an in vitro co-culture model with human intestinal epithelial cells (IECs) and human blood leukocytes has been developed to assess ion transport and barrier function (McKay et al., 1996), or to investigate whether reactivity of enterocytes to bacterial signals depends on their interactions with immunocompetent cells (Haller et al., 2000). In addition, both human peripheral blood mononuclear cells (PBMCs) and lamina propria mononuclear cells are involved in the mucosal defence against bacterial translocation and systemic inflammation (Guzy et al., 2009). Therefore, PBMCs in the basolateral compartment of Transwell plates can be in vitro co-cultured with apical IECs to evaluate the effect of bacteria on immunological interactions between these two main cell populations in the gut.

In the past decade, the clinical implications and immunomodulatory effects of probiotic bacteria, such as lactobacilli, have been increasingly investigated in both clinical trials and experimental models. In clinical practice, commercial probiotics have been used as therapeutic agents to ameliorate diarrhoea. Treatment with Lactobacillus rhamnosus strains can shorten the duration of diarrhoea up to 5 days in patients with rotavirus infections (Szymanski et al., 2006). On the other hand, some in vitro studies showed that lactobacilli can not only blunt the pro-inflammatory immune response induced by Salmonella enterica serotype Typhimurium ( $S$. Typhimurium) in HT-29 human IECs (O'Hara et al., 2006), but also inhibit the synthesis of interleukin-8 (IL-8) in Salmonella-stimulated Caco-2 cells (Nemeth et al., 2006). However, long-duration kinetic impacts of probiotic lactobacilli on pro-inflammatory cytokine responses and integrity of intestinal barrier function after pathogenic stimulation in an enterocyte-leukocyte co-culture model has rarely been discussed. Thus, we conducted this study to assess the inhibitory effects of the commercial probiotic Lactobacillus casei subsp. rhamnosus (Lcr35), which has been used as a probiotic therapeutic agent (Fang et al., 2009), upon Salmonella LPS-induced inflammation and epithelial barrier dysfunction in IECs over a $48 \mathrm{~h}$ period in vitro.

\section{METHODS}

Bacterial strain and cultivation. The commercial strain Lactobacillus casei subsp. rhamnosus, Lcr35 (Antibiophilus), was used in this study. Lcr35 was cultured in Difco Lactobacilli MRS (de ManRogosa-Sharpe) broth (Becton Dickinson) at $37{ }^{\circ} \mathrm{C}$ with $5 \% \mathrm{CO}_{2}$ and used in the early stationary phase.

Cell culture. Enterocyte-like Caco- 2 cells with the structural and functional characteristics of intestinal cells were developed into differentiated intestinal cells (Koninkx et al., 1996). The human Caco2 cells (passage 33-35) were purchased from the Cell Bank at the Bioresource Collection and Research Centre (Hsinchu, Taiwan), seeded at a density of $1 \times 10^{5}$ cells $\mathrm{cm}^{-2}$ and grown on $0.9 \mathrm{~cm}^{2}$ cell culture inserts in the Transwell system $(0.4 \mu \mathrm{m}$ nucleopore size BD Falcon; Milian). The inserts were pre-coated with collagen (Sigma) and placed into 12-well tissue culture plates before cell seeding. Cells were maintained in Dulbecco's modified Eagle medium (DMEM, $4500 \mathrm{mg}$ glucose $1^{-1}$; Amimed) with $20 \%$ (v/v) fetal bovine serum (FBS) and $1 \%(\mathrm{v} / \mathrm{v})$ non-essential amino acids (Sigma). Cell culture medium was changed every 2-3 days until 12-14 days later when full polarization of the Caco-2 cell monolayer was achieved.

Isolation of PBMCs. The study protocol and consent documents were approved by the Institutional Review Board of Mackay Memorial Hospital. PBMCs were obtained from healthy donors and provided as buffy coats (Blood Transfusion Centre, Taipei, Taiwan). Leukocytes were freshly purified by Ficoll-Hypaque (Pharmacia) centrifugation $(400 \mathrm{~g}, 20 \mathrm{~min})$ and were collected from the interface. The final PBMCs were stored in RPMI 1640 medium (Sigma), supplemented with $1.0 \mathrm{mM}$ HEPES, $1.0 \mathrm{mM}$ sodium pyruvate, $0.05 \mathrm{mM}$ 2-mercaptoethanol, $10 \%(\mathrm{v} / \mathrm{v})$ FBS and adjusted to $3 \times 10^{6}$ cells $\mathrm{ml}^{-1}$.

LPS stimulation of Caco-2/PBMCs in the co-culture model. Confluent monolayers of polarized Caco-2 cells were developed in tissue culture inserts and transferred to 12 -well tissue culture plates. In the Transwell cell culture system, $1 \mathrm{ml}$ fresh purified PBMCs and $1 \mathrm{ml}$ DMEM, respectively, were added to the basal and apical compartments of the culture plates. The apical surface of Caco-2 cell monolayers was challenged with $S$. Typhimurium LPS (Sigma), at the optimal concentration of $10 \mathrm{ng} \mathrm{ml}^{-1}$, to activate maximal cytokine tumour necrosis factor alpha (TNF- $\alpha$ ) secretion (Ménard et al., 2004). In order to trigger an inflammatory response in the whole co-culture system, the Caco- 2 cell monolayers were incubated with LPS at $37{ }^{\circ} \mathrm{C}$ with $5 \% \mathrm{CO}_{2}$ for $48 \mathrm{~h}$ (Matsumoto \& Benno, 2006). After then, the Caco-2 cells and PBMCs were collected and washed once with cold PBS (Fig. 1). After centrifugation ( $300 \mathrm{~g}, 5 \mathrm{~min}$ ), the washed PBMCs were resuspended in the supplemented RPMI 1640 medium, and then were added back to the original basal compartments in the Transwell plates for further experiments (Fig. 1).

Probiotic anti-inflammatory effects on intestinal cells in vitro. To investigate the probiotic anti-inflammatory effects on Caco- 2 cells, an inoculum of $2 \times 10^{7}$ c.f.u. Lcr35 $\mathrm{ml}^{-1}$ was added onto the apical side of enterocytes after removal of LPS (Fig. 1). The cell survival was assessed using a 3-(4,5-dimethyl-thiazol-2-y1)-2,5-diphenyltetrazolium bromide assay (Matsumoto \& Benno, 2006). Compared to the untreated controls, the relative survival rate of polarized Caco- 2 cells treated with Lcr35 was still maintained (>95\%) after 48 h. Caco-2/ PBMCs co-cultures were incubated for $1,6,24$ or $48 \mathrm{~h}$. The immune responses of Caco-2 cells and PBMCs upon stimulation with Lcr35 were monitored by analysis of cytokine gene transcription including IL-8, monocyte chemoattractant protein 1 (MCP-1) and transforming growth factor- $\beta 1$ (TGF- $\beta 1$ ) using RT-PCR, and by determination of IL-8 secretion in cell culture supernatants using ELISA. In all coculture experiments, IL- 8 protein levels were measured in both the 


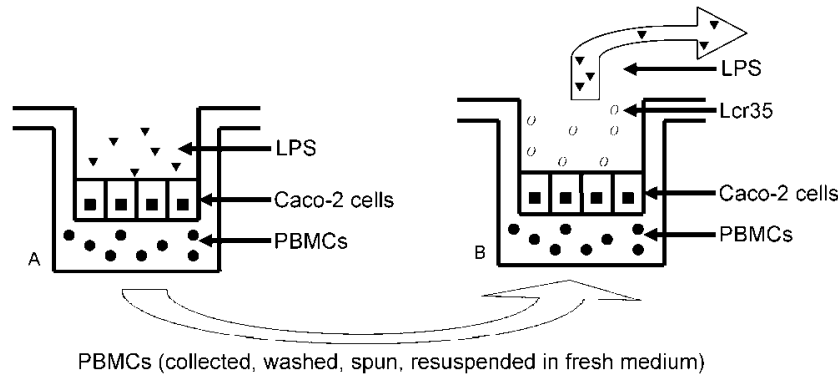

Fig. 1. Schematic illustration of the co-culture model showing experimental arrangements in the Transwell system for assessment of Caco-2/PBMCs interactions after various kinds of stimulation: inflammation (A), Caco-2 cells were challenged with LPS; antiinflammation (B), LPS was removed and then Caco-2 cells were challenged with Lcr35 with the same population of PBMCs washed, spun, resuspended in complemented RPMI medium, and reloaded into the lower chamber.

apical and basal media, which were collected by centrifugation at 2000 r.p.m. for $3 \mathrm{~min}$ and frozen in aliquots at $-80{ }^{\circ} \mathrm{C}$. IL-8 protein levels were quantified by ELISA using the DuoSet kit (R\&D Systems) according to the manufacturer's instructions. Absorbance was measured in a microplate reader (SpectraMax 190; Molecular Devices) at $450 \mathrm{~nm}$.

RNA extraction and PCR. Total RNA from Caco-2 cells was isolated using the RNA extraction miniprep system (Total RNA Mini kit; Viogene) according to the manufacturer's instructions for animal cells. Template cDNA was synthesized from RNA using reverse transcription with Oligo $\mathrm{dT}_{12-18}$ primers (Protech) and amplified in a total volume of $50 \mu \mathrm{l}$ reaction using Taq DNA polymerase (Genet Bio) in the GeneAmp PCR System 9700 (Applied Biosystems) with 40 cycles of denaturation at $94{ }^{\circ} \mathrm{C}$ for $30 \mathrm{~s}$, annealing at $56{ }^{\circ} \mathrm{C}$ (actin and MCP-1) or $63{ }^{\circ} \mathrm{C}$ (IL-8 and TGF- $\beta 1$ ) for $30 \mathrm{~s}$, and extension at $72{ }^{\circ} \mathrm{C}$ for $30 \mathrm{~s}$. Subsequently, the PCR products were subjected to gel electrophoresis on $2 \%$ agarose gels with $1 \times$ Tris borate EDTA and visualized by staining with ethidium bromide. Pairs of oligonucleotide primers specific to IL-8 (forward 5' -TGGCTCTCTTGGCAGCCTTC-3', reverse $3^{\prime}$-TGCACCCAGTTTTCCTTGGG-5'; product length $238 \mathrm{bp}$ ), MCP-1 (forward 5'-CTCAGCCAGATGCAATCAAT-3', reverse 3'-AGATTCTTGGGTTGTGGAGT-5'; 259 bp), and TGF- $\beta 1$ (forward $5^{\prime}$-AGGACTGCGGATCTCTGTGT-3', reverse $3^{\prime}$ AGTGCCCAAGGTGCTCAATA-5'; 196 bp) were used as described by Saegusa et al. (2007) and purchased from Invitrogen. Primer sequence of the housekeeping gene actin (forward 5' -GTCACCAACTGGGACGACAT-3', reverse 3'-GATAGCAGCCTGGATAGCAA-5'; $197 \mathrm{bp}$ ) was designed by the primer 5 program. The intensity of the specific bands was measured using the Multi Gauge v2.1 image software.

Electrical resistance measurements. Transepithelial electrical resistance (TEER) of Caco-2 cells was monitored using an EVOM TEER meter (World Precision Instruments). TEER increased until day 12-14 when a steady state of higher than $450 \Omega \mathrm{cm}^{2}$ was reached, indicating development of functional polarity and an intact monolayer, which is referred to as completed tight junction formation and maximized integrity of barrier function, and is similar to that described by Klingberg et al. (2005), Mine \& Zhang (2003) and Satsu et al. (2003). LPS stimulation of Caco-2/PBMCs in the co-culture model lasted for $48 \mathrm{~h}$ until addition of Lcr35. After removal of LPS by washing the Caco-2 monolayer with PBS, TEER readings were recorded at five time points $(0,1,6,24$ and $48 \mathrm{~h})$ during the subsequent co-incubation of Caco-2 and Lcr35 $\left(2 \times 10^{7}\right.$ c.f.u. $\left.\mathrm{ml}^{-1}\right)$. An m.o.i. of 25 was calculated when Lcr35 was inoculated.

Statistics. The results were obtained using PBMCs from at least three blood donors in each independent experiment. The quantitative data were expressed as mean \pm SEM of triplicate measurements. Statistical analyses were performed with one-way ANOVA or Student's $t$-test using SPSS 12.0. Statistical significance was defined as a $P$ value of $<0.05$.

\section{RESULTS AND DISCUSSION}

\section{Salmonella LPS-induced inflammation}

Our pilot study demonstrated that IL-8 levels in the apical and basal compartment of Caco-2/PBMCs co-culture system were, respectively, $838 \mathrm{pg} \mathrm{ml}^{-1}(P>0.05)$ and $7046562 \mathrm{pg} \mathrm{ml}^{-1}(P<0.001)$ after Salmonella LPS challenge for $48 \mathrm{~h}$, compared to 703 and $6729 \mathrm{pg} \mathrm{ml}^{-1}$, respectively, in non-LPS controls. In the present study, Salmonella LPS caused a significant inflammatory response, which was prolonged as late as $6-24 \mathrm{~h}$ after removal of the LPS, in polarized Caco-2 cells by triggering the mRNA expression of three cytokine genes, including IL-8 ( 0 vs 1,6 and 24 h, Fig. 2). Both IL-8 gene and its post-transcriptional protein expression validate that Salmonella LPS can induce an inflammation in our Caco2/PBMCs co-culture system. Previous reports demonstrated that Salmonella (Bolton et al., 2000), or its cell wall components (Sierro et al., 2001), may participate in initiating the mucosal inflammation of Caco-2 cells. LPS interacts with the intestinal epithelium and indirectly induces the inflammatory responses of the lymphoid system. Moreover, LPS has been reported to moderately enhance IL-8 secretion in Caco-2 cells (Chen et al., 2006; Weglarz et al., 2007). Such an LPS challenge to Caco-2 cells can simulate Salmonella enteric infections in vivo because increased IL-8 secretion triggers recruitment of neutrophils to intestinal epithelium, which is the histopathological hallmark of salmonellosis (McCormick et al., 1993).

\section{IL-8 secretion in the presence of Lcr35}

Our preliminary studies indicated that the IL- 8 secretion in the apical compartment was lower than that in the basal compartment in the Caco-2/PBMCs co-cultures, and also was negligible in the Caco-2 cells without co-cultured PBMCs (data not shown). In a similar Transwell system, the relevant cytokines were only detectable in the basolateral component (Haller et al., 2000). Therefore, the IL-8 concentration was exclusively measured from the medium in the basal compartment. Our data showed that the inhibition of IL-8 levels by Lcr35 was initially apparent at $24 \mathrm{~h}$, and the trend reached its maximum at $48 \mathrm{~h}$ (Fig. $3)$. The IL-8 levels in the medium from Lcr35-treated cells were lower than those from non-Lcr35-treated cells at $48 \mathrm{~h}$ $(P<0.05)$. 


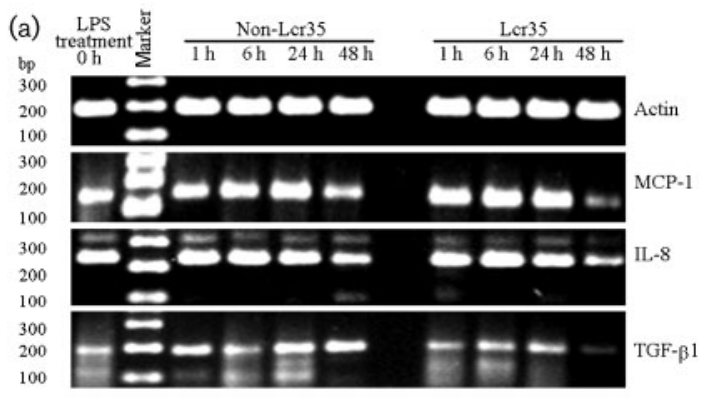

(b)
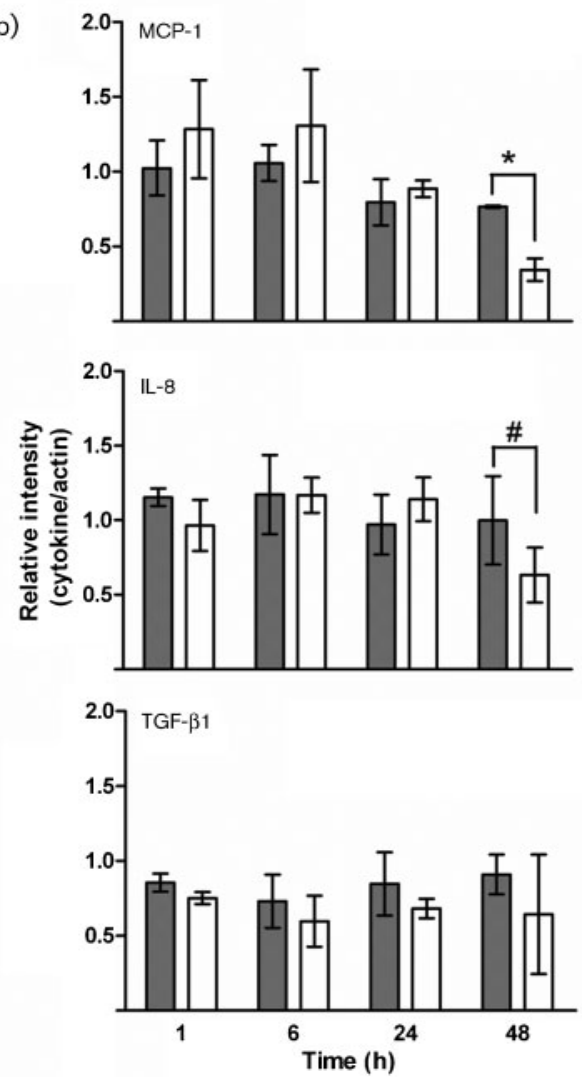

Fig. 2. mRNA expression of various cytokines (MCP-1, IL-8 and TGF- $\beta 1$ ) in Caco-2 cells after treatment with Lcr35 for 1, 6, 24 and 48 h. Caco-2/PBMCs were pre-incubated with Salmonella LPS for $48 \mathrm{~h}$. (a) Representative PCR showing identified bands accompanied by 100 bp DNA ladder markers. (b) Quantification of mRNA expression in MCP-1, IL-8 and TGF- $\beta 1$, converted into relative intensity of individual PCR bands, was compared after normalization using the housekeeping gene actin. ${ }^{*}, P<0.05$ indicates a significant difference between the Lcr35-treated group and the control group (Student's $t$-test, $n=3$ ); \#, $P=0.05$. Grey bars, Non-Lcr35; white bars, Lcr35.

A putative mechanism of the aforementioned phenomenon is that probiotic bacteria could either bind to cell surface receptors on enterocytes, or stimulate the translocation of dendritic cells or secrete mediators diffusing into the cytosols of enterocytes triggering the related signal transduction pathways (Koninkx et al., 1996). IL-8

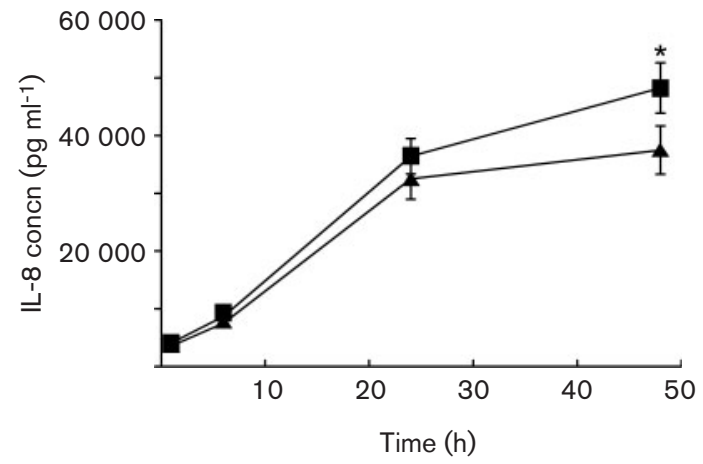

Fig. 3. Lcr35 inhibited IL-8 secretion in the Caco-2/PBMCs coculture model after LPS-induced inflammatory responses for $48 \mathrm{~h}$. Secretion of IL-8 into the basolateral compartment was determined at various time points (1, 6, 24 and $48 \mathrm{~h})$. Culture medium from non-Lcr35-treated cells was used as a control. The data are expressed as means \pm SEM (error bars) of measurements in triplicate. ${ }^{*}, P<0.05$ indicates a significant difference between the Lcr35-treated group and the control group (one-way ANOVA, $n=3)$. $\mathbf{\square}$, Non-Lcr35; $\boldsymbol{\Delta}$, Lcr35.

secretion can be induced through the nuclear factor-kappa $\mathrm{B}(\mathrm{NF}-\kappa \mathrm{B})$ pathway after challenge of TNF- $\alpha$ or pathogenassociated molecular patterns (PAMPs), such as LPS and flagellin. The signalling pathway of NF- $\kappa \mathrm{B}$ activation after Shigella flexneri infection involves the inhibitory protein, I$\kappa \mathrm{B} \alpha$, which can be upregulated by $L$. casei to block NF- $\kappa \mathrm{B}$ activation in Caco-2 cells (Tien et al., 2006). Probiotic Escherichia coli strain Nissle 1917 suppresses TNF- $\alpha-$ induced IL-8 production in human colonic epithelial cells (Kamada et al., 2008). The secretion of IL-8 in the apical compartment was low in both Lcr35-treated and nonLcr35-treated cells in our study. After Lcr35 treatment, IL-8 secretion was obviously reduced in the basolateral compartment with time compared to the controls. The immune response in Caco-2 cells could be further enhanced by presence of PBMCs after adequate interaction. Furthermore, the inhibition of IL-8 secretion demonstrated that Lcr35 is able to suppress the inflammatory response in vitro.

\section{mRNA expression in Caco-2/PBMCs co-cultures with Lcr35}

Our preliminary experiment confirmed that IL-8 mRNA expression was strongly increased by LPS stimulation for $48 \mathrm{~h}$, accompanied by corresponding increased IL- 8 protein secretion (data not shown). In this study, the LPS-stimulated polarized Caco-2 monolayers, treated with or without Lcr35 for different periods of time, showed that the bands of PCR-amplified IL-8, TGF- $\beta 1$ and MCP- 1 were similarly maintained in their density before $24 \mathrm{~h}$, but only MCP-1 obviously decreased after $48 \mathrm{~h}$ after inoculation of Lcr35 (Fig. 2). First, mRNA expression of MCP-1 in the 
presence of Lcr35 was significantly lower than non-Lcr35 controls at the $48 \mathrm{~h}$ time point $(P<0.05$, Fig. 2$)$. Second, incubation of Caco-2 cells with Lcr35 for $48 \mathrm{~h}$ showed a decreasing trend of IL-8 mRNA expression $(P=0.05)$. Third, there was no significant difference in mRNA expression of TGF- $\beta 1$ between Lcr35-treated cells and non-Lcr35 controls.

In this study, we used a simplified epithelial-immune cell co-culture system to investigate the anti-inflammatory effects of probiotic Lcr35 by analysis of gene expression in the inflamed IECs. The results demonstrated that IL-8, a chemoattractant for neutrophils (Huber et al., 1991), was significantly downregulated by Lcr35 in the inflamed Caco2 cells after $48 \mathrm{~h}$ duration, but not within $24 \mathrm{~h}$. Similarly, Lactobacillus rhamnosus GG can decrease IL-8 production in TNF- $\alpha$ stimulated Caco- 2 cells (Zhang et al., 2005). In addition, TGF- $\beta 1$ is an important inhibitor of the synthesis of pro-inflammatory cytokines (Parlesak et al., 2004). In the present study, Lcr35 did not significantly affect TGF- $\beta 1$ gene expression in the inflamed Caco- 2 cells over $48 \mathrm{~h}$. Hence, TGF- $\beta 1$ might not play the key role of inhibiting IL-8 secretion of Caco-2/PBMCs after Salmonella LPS challenge. Interestingly, a previous study revealed that Lactobacillus johnsonii stimulated TGF- $\beta 1$ mRNA expression but Lactobacillus sakei did not in the Caco-2/PBMCs co-culture model (Haller et al., 2000). Thus, various strains of lactobacilli may exert different regulation upon TGF- $\beta 1$ gene expression. Moreover, inflammatory stimuli can induce MCP-1, which is a potent chemoattractant for monocytes, memory $\mathrm{T}$ lymphocytes and natural killer cells (Gu et al., 2000). Probiotic E. coli Nissle 1917 specifically upregulated MCP-1 mRNA expression in Caco-2 cells within $6 \mathrm{~h}$ (Ukena et al., 2005); this is similar to our finding of increasing band density of PCR-amplified MCP1 mRNA within $1 \mathrm{~h}$ (Fig. 2). In contrast, L. sakei and nonpathogenic E. coli upregulated MCP-1 gene expression in Caco-2/PBMCs co-cultures at $16 \mathrm{~h}$ (Haller et al., 2000). These data suggested that Lcr35 might initially stimulate host defence mechanisms via the expression of MCP-1 at an earlier stage than other probiotic strains. Strain specificity of lactobacilli or co-existence of PBMCs might explain the varied dynamics in upregulation of MCP-1 gene expression. All of these studies indicated that cytokines or chemokines might be secreted from IECs. Therefore, alteration in gene expression of various cytokines or chemokines in Caco-2 cells could result in subsequent variation of individual protein expression and potentially interact with PBMCs to induce appropriate host immune responses.

\section{Effect of Lcr35 on integrity of Caco-2 monolayer}

In the treatment of intestinal inflammatory disorders it is fundamental to restore and stabilize physical barrier function. Probiotics have been reported to strengthen gut barrier function (Klingberg et al., 2005). During the first $48 \mathrm{~h}$ period in our Caco-2/PBMCs co-cultures, TEER decreased from 420.7 to $414.0 \Omega \mathrm{cm}^{2}$ in LPS-treated Caco-2 cells but increased from 420.7 to $455.0 \Omega \mathrm{cm}^{2}$ in non-LPStreated cells $(P<0.05)$. To determine the effect of Lcr35 on LPS-induced epithelial barrier dysfunction of Caco-2 cells, TEER was monitored for $48 \mathrm{~h}$ and this showed a significant reinforcement by Lcr35 of the TEER of the Caco-2 cell monolayers with time. Compared to the controls, the TEER of the Lcr35-treated cells increased by $12.1 \%$ (598.8 vs $\left.534.2 \Omega \mathrm{cm}^{2}, P<0.001\right)$ after $1 \mathrm{~h}$ and by $5.7 \%(517.7$ vs $\left.489.7 \Omega \mathrm{cm}^{2}, P<0.05\right)$ after $6 \mathrm{~h}$. The TEER levels of the Lcr35-treated Caco-2 cells were $86.7 \%$ higher than those of the non-Lcr35-treated cells ( 874 vs $468 \Omega \mathrm{cm}^{2}, P<0.001$ ) at $48 \mathrm{~h}$ (Fig. 4). Such a biphasic booster effect of Lcr35 on the TEER of the Caco-2 monolayers appeared firstly at $1 \mathrm{~h}$ with an increase of $64.6 \Omega \mathrm{cm}^{2}$, followed by another second surge of $406 \Omega \mathrm{cm}^{2}$ at $48 \mathrm{~h}$ when compared to non-Lcr35treated cells (Fig. 4). In this time-dependent phenomenon, withdrawal of LPS and replacement of the medium might account for the first peak; competitive consumption of nutrients in the medium by apical Lcr35 and basal PBMCs might temporarily reduce TEER between 1 and $24 \mathrm{~h}$, but accumulation of released probiotic mediators or their influences upon Caco-2/PBMCs co-cultures taking longer (than $24 \mathrm{~h}$ ) might exceed the above transitory decrease in TEER to form the second higher peak at $48 \mathrm{~h}$. TEER of polarized Caco-2 monolayers exposed to Lactobacillus salivarius DC5 and Lactobacillus plantarum MF1298 also showed a similar trend (Klingberg et al., 2005). Unlike employing probiotic lactobacilli as a post-infectious therapeutic agent in our experiment, in a different study another probiotic mixture, VSL\#3, was applied to prevent a pathogen-induced decrease in TEER (Otte \& Podolsky, 2004). To sum up, Lcr35 can retain or even enhance the TEER of Caco-2 cell monolayers, which validates its

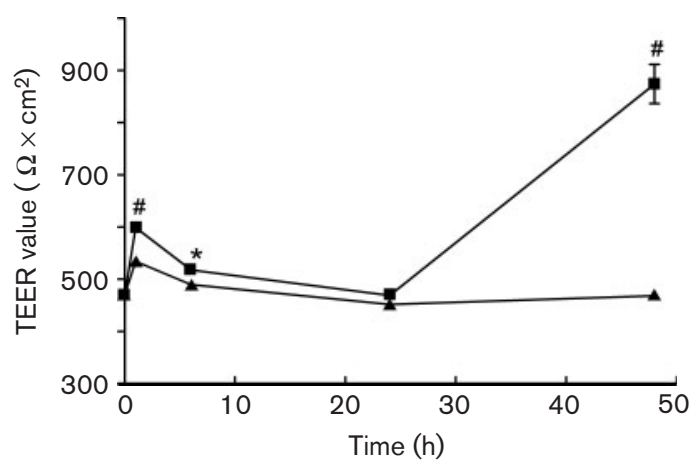

Fig. 4. Lcr35 increased TEER levels of the Caco-2 cell monolayers. The cell monolayers were pre-stimulated with Salmonella LPS for $48 \mathrm{~h}$, followed by treatment with Lcr35 that reinforced LPS-suppressed transepithelial resistance. TEER values were measured at various time points $(1,6,24$ and $48 \mathrm{~h}$ ) and the data are expressed as means \pm SEM (error bars) of measurements in triplicate. * $P<0.05$ indicates significant difference between the Lcr35-treated group and the control group (one-way ANOVA, $n=3$ ); \#, $P<0.001$. $\mathbf{\square}$, Non-Lcr35; $\mathbf{\Delta}$, Lcr35. 
beneficial role as a post-infectious therapeutic agent in the maintenance and reinforcement of intestinal barrier integrity. This finding is supported by the fact that several probiotics and commensal bacteria, including L. rhamnosus GG and Bifidobacterium infantis, have been shown to stabilize tight junction-associated protein, zonula occludens-1, or increased zonula occludens-1 expression (Ewaschuk et al., 2008; Johnson-Henry et al., 2008) so as to strengthen epithelial barrier function. Thus, judging from the overall results of inhibition in IL-8 secretion, altered gene expression of MCP-1 and enhancement of TEER in the Caco-2 cell monolayers in our study, Lcr35 could post-infectiously inhibit inflammation of enterocytes caused by enteric pathogens through a putative mechanism involving the integrity of the tight junctions between enterocytes. In other words, Lcr35 functions as a barrier-sustaining agent, or barrier-enhancing agent, to protect the breakdown of intestinal epithelium from enteric pathogens.

In conclusion, our study using the Caco-2/PBMCs coculture model clearly demonstrated the pivotal role of Lcr35 in inhibition of the intestinal anti-inflammatory responses and its beneficial effect upon re-establishment of the intestinal epithelial barrier function after inflammation. Moreover, Lcr35 induced the Caco-2 cells to express various pro-inflammatory cytokines at the gene level. Whether Lcr35 could further signal the PBMCs after such gene regulation to mediate the subsequent responses needs further studies for elucidation. Upon the inhibitory effect of probiotic Lcr35, the secretion of IL-8, a major pathogenelicited epithelial chemoattractant of the inflammatory responses in salmonellosis, was significantly reduced in the basolateral compartment of a co-culture model where PBMCs predominantly resided and mimicked lymphocytes in the lamina propria. PBMCs have been used elsewhere to study the effect of immunocompetent cells on human IECs (Haller et al., 2000; McKay et al., 1996). Hence, our proposed Caco-2/PBMCs co-culture model is not only a useful in vitro system for investigating the modulation of probiotics on immune responses and barrier integrity of the gut after enteric infection or inflammation, but also a feasible platform for screening probiotic therapeutic agents for further animal studies and clinical trials.

\section{ACKNOWLEDGEMENTS}

This study was financially supported by Mackay Memorial Hospital, National Taipei University of Technology (NTUT 97-140-3), and GenMont Biotech Incorporation, Taiwan.

\section{REFERENCES}

Bolton, A. J., Osborne, M. P. \& Stephen, J. (2000). Comparative study of the invasiveness of Salmonella serotypes Typhimurium, Choleraesuis and Dublin for Caco-2 cells, HEp-2 cells and rabbit ileal epithelia. J Med Microbiol 49, 503-511.

Chen, J., Ng, C. P., Rowlands, D. K., Xu, P. H., Gao, J. Y., Chung, Y. W. \& Chan, H. C. (2006). Interaction between enteric epithelial cells and
Peyer's patch lymphocytes in response to Shigella lipopolysaccharide: effect on nitric oxide and IL-6 release. World J Gastroenterol 12, 38953900.

Ewaschuk, J. B., Diaz, H., Meddings, L., Diederichs, B., Dmytrash, A., Backer, J., Looijer-van Langen, M. \& Madsen, K. L. (2008). Secreted bioactive factors from Bifidobacterium infantis enhance epithelial cell barrier function. Am J Physiol Gastrointest Liver Physiol 295, G1025G1034.

Fang, S. B., Lee, H. C., Hu, J. J., Hou, S. Y., Liu, H. L. \& Fang, H. W. (2009). Dose-dependent effect of Lactobacillus rhamnosus on quantitative reduction of faecal rotavirus shedding in children. J Trop Pediatr 55, 297-301.

Gu, L., Tseng, S., Horner, R. M., Tam, C., Loda, M. \& Rollins, B. J. (2000). Control of TH2 polarization by the chemokine monocyte chemoattractant protein-1. Nature 404, 407-411.

Guzy, C., Schirbel, A., Paclik, D., Wiedenmann, B., Dignass, A. \& Sturm, A. (2009). Enteral and parenteral nutrition distinctively modulate intestinal permeability and $\mathrm{T}$ cell function in vitro. Eur $\mathrm{J}$ Nutr 48, 12-21.

Haller, D., Bode, C., Hammes, W. P., Pfeifer, A. M., Schiffrin, E. J. \& Blum, S. (2000). Non-pathogenic bacteria elicit a differential cytokine response by intestinal epithelial cell/leucocyte co-cultures. Gut 47, 79-87.

Huber, A. R., Kunkel, S. L., Todd, R. F., III \& Weiss, S. J. (1991). Regulation of transendothelial neutrophil migration by endogenous interleukin-8. Science 254, 99-102.

Johnson-Henry, K. C., Donato, K. A., Shen-Tu, G., Gordanpour, M. \& Sherman, P. M. (2008). Lactobacillus rhamnosus strain GG prevents enterohemorrhagic Escherichia coli O157:H7-induced changes in epithelial barrier function. Infect Immun 76, 1340-1348.

Kamada, N., Maeda, K., Inoue, N., Hisamatsu, T., Okamoto, S., Hong, K. S., Yamada, T., Watanabe, N., Tsuchimoto, K. \& other authors (2008). Nonpathogenic Escherichia coli strain Nissle 1917 inhibits signal transduction in intestinal epithelial cells. Infect Immun 76, 214-220.

Klingberg, T. D., Pedersen, M. H., Cencic, A. \& Budde, B. B. (2005). Application of measurements of transepithelial electrical resistance of intestinal epithelial cell monolayers to evaluate probiotic activity. Appl Environ Microbiol 71, 7528-7530.

Koninkx, J. F., Brown, D. S., Kok, W., Hendriks, H. G., Pusztai, A. \& Bardocz, S. (1996). Polyamine metabolism of enterocyte-like Caco-2 cells after exposure to Phaseolus vulgaris lectin. Gut 38, 47-52.

Matsumoto, M. \& Benno, Y. (2006). Anti-inflammatory metabolite production in the gut from the consumption of probiotic yogurt containing Bifidobacterium animalis subsp. lactis LKM512. Biosci Biotechnol Biochem 70, 1287-1292.

McCormick, B. A., Colgan, S. P., Delp-Archer, C., Miller, S. I. \& Madara, J. L. (1993). Salmonella typhimurium attachment to human intestinal epithelial monolayers: transcellular signalling to subepithelial neutrophils. J Cell Biol 123, 895-907.

McKay, D. M., Croitoru, K. \& Perdue, M. H. (1996). T cell-monocyte interactions regulate epithelial physiology in a coculture model of inflammation. Am J Physiol 270, C418-C428.

Ménard, S., Candalh, C., Bambou, J. C., Terpend, K., CerfBensussan, N. \& Heyman, M. (2004). Lactic acid bacteria secrete metabolites retaining anti-inflammatory properties after intestinal transport. Gut 53, 821-828.

Mine, Y. \& Zhang, J. W. (2003). Surfactants enhance the tight-junction permeability of food allergens in human intestinal epithelial Caco-2 cells. Int Arch Allergy Immunol 130, 135-142.

Mitic, L. L., Van Itallie, C. M. \& Anderson, J. M. (2000). Molecular physiology and pathophysiology of tight junctions I. Tight junction structure and function: lessons from mutant animals and proteins. Am J Physiol Gastrointest Liver Physiol 279, G250-G254. 
Nemeth, E., Fajdiga, S., Malago, J., Koninkx, J., Tooten, P. \& van Dijk, J. (2006). Inhibition of Salmonella-induced IL-8 synthesis and expression of Hsp70 in enterocyte-like Caco-2 cells after exposure to non-starter lactobacilli. Int J Food Microbiol 112, 266-274.

O'Hara, J. R. \& Buret, A. G. (2008). Mechanisms of intestinal tight junctional disruption during infection. Front Biosci 13, 7008-7021.

O'Hara, A. M., O'Regan, P., Fanning, A., O'Mahony, C., Macsharry, J., Lyons, A., Bienenstock, J., O'Mahony, L. \& Shanahan, F. (2006). Functional modulation of human intestinal epithelial cell responses by Bifidobacterium infantis and Lactobacillus salivarius. Immunology 118, 202-215.

Otte, J. M. \& Podolsky, D. K. (2004). Functional modulation of enterocytes by gram-positive and gram-negative microorganisms. Am J Physiol Gastrointest Liver Physiol 286, G613-G626.

Parlesak, A., Haller, D., Brinz, S., Baeuerlein, A. \& Bode, C. (2004). Modulation of cytokine release by differentiated CACO-2 cells in a compartmentalized coculture model with mononuclear leucocytes and nonpathogenic bacteria. Scand J Immunol 60, 477-485.

Ramiro-Puig, E., Perez-Cano, F. J., Castellote, C., Franch, A. \& Castell, M. (2008). The bowel: a key component of the immune system. Rev Esp Enferm Dig 100, 29-34.

Saegusa, S., Totsuka, M., Kaminogawa, S. \& Hosoi, T. (2007). Cytokine responses of intestinal epithelial-like Caco-2 cells to nonpathogenic and opportunistic pathogenic yeasts in the presence of butyric acid. Biosci Biotechnol Biochem 71, 2428-2434.

Satsu, H., Yokoyama, T., Ogawa, N., Fujiwara-Hatano, Y. \& Shimizu, M. (2003). Effect of neuronal PC12 cells on the functional properties of intestinal epithelial Caco-2 cells. Biosci Biotechnol Biochem 67, 13121318.

Sierro, F., Dubois, B., Coste, A., Kaiserlian, D., Kraehenbuhl, J. P. \& Sirard, J. C. (2001). Flagellin stimulation of intestinal epithelial cells triggers CCL20-mediated migration of dendritic cells. Proc Natl Acad Sci U S A 98, 13722-13727.

Szymanski, H., Pejcz, J., Jawien, M., Chmielarczyk, A., Strus, M. \& Heczko, P. B. (2006). Treatment of acute infectious diarrhoea in infants and children with a mixture of three Lactobacillus rhamnosus strains - a randomized, double-blind, placebo-controlled trial. Aliment Pharmacol Ther 23, 247-253.

Tien, M. T., Girardin, S. E., Regnault, B., Le, B. L., Dillies, M. A., Coppee, J. Y., Bourdet-Sicard, R., Sansonetti, P. J. \& Pédron, T. (2006). Anti-inflammatory effect of Lactobacillus casei on Shigellainfected human intestinal epithelial cells. J Immunol 176, 1228-1237.

Ukena, S. N., Westendorf, A. M., Hansen, W., Rohde, M., Geffers, R., Coldewey, S., Suerbaum, S., Buer, J. \& Gunzer, F. (2005). The host response to the probiotic Escherichia coli strain Nissle 1917: specific up-regulation of the proinflammatory chemokine MCP-1. BMC Med Genet 6, 43.

Weglarz, L., Wawszczyk, J., Orchel, A., Jaworska-Kik, M. \& Dzierzewicz, Z. (2007). Phytic acid modulates in vitro IL-8 and IL6 release from colonic epithelial cells stimulated with LPS and IL-1 $\beta$. Dig Dis Sci 52, 93-102.

Zhang, L., Li, N., Caicedo, R. \& Neu, J. (2005). Alive and dead Lactobacillus rhamnosus GG decrease tumor necrosis factor- $\alpha$-induced interleukin-8 production in Caco-2 cells. J Nutr 135, 1752-1756. 